Our Nature (2003) 1

\begin{tabular}{|c|l|l|l|c|c|}
\hline 71 & Common Rosefinch & Carpodacus erythrinus & Fringillidae & - & ST \\
\hline 72 & Red-headed Bullfinch & Pyrrhula erythrocephala & $"$ & 1 & D \\
\hline 73 & Little Bunting & Emberiza pusilla & Emberizidae & 2 & OTS \\
\hline
\end{tabular}

$\begin{array}{llllll}\text { Note: } & & & & \\ 1 & = & \text { Common TO } & = & \text { Thin / Open } \\ 2 & = & \text { Fairly common } & \mathrm{T} & = & \text { Thin } \\ 3 & = & \text { Occasional } & \text { ST } & = & \text { Scrub / Thin } \\ 4 & = & \text { Uncommon } & \text { D } & = & \text { Dense } \\ 5 & = & \text { Rare } & \text { OTS } & & \text { Open / Thin / Scrub } \\ \mathrm{S} 1 & = & \text { Status } & & & \\ + & = & \text { Internationally significant breeding population }\end{array}$

Mala Rai

Central Department of Zoology Tribhuvan University, Kathmandu Corresondence: Department of Biology, Central Campus of Technology, Hattisar, Dharan

\section{References}

Ali, S. and S. D. Ripley 1995. A Pictorial Guide to the Birds of Indian Subcontinent. Bombay Natural History Society, Oxford University Press, Bombay

Fleming, R. L (Sr)., R. L. Fleming (Jr) and L. S. Bangel 1979. Birds of Nepal, Second Edition, Avalok Publishers, Kathmandu, Nepal
Inskipp, C and T. Inskipp 1985. A Guide to the Birds of Nepal. Croom Helm, London, England

Rai, M. 1998. The impact of development activities on Avifauna of Tamor river basin" M. Sc. Thesis, Central Department of Zoology, T. U., Kirtipur Kathmandu.

\title{
3. Birds of Itahari
}

Itahari municipality is one of the three municipalities of Sunsari district. It is situated in the Tarai region of Eastern part of Nepal. The main rivers are Sunsari, Tengra and Budhi. Besides that there are also few man-made and natural wetlands. Itahari is very near from the mixed type of jungle popularly known as 'Charkoshe Jhadi' on the north, which provides ideal shelter for the birds. This is the reason why many local migratory birds as well as other birds are found to come very commonly from the jungle.
It was the month of November 2000 when I went to Itahari to watch birds for the first time. Then I kept watching the birds at that place regularly till January 2001. During that period I visited different sites several times at least once a week regularly and also collected information from the locals.

The present report is the result of a study of about 14 months. Regular observations and local enquiry were also made. I tried my best to identify the birds correctly using available literature (Ali 
Our Nature (2003) 1

and Ripley 1984, Fleming et al.1984, Ali 1989, Ali 1990, Subba 1995a, Subba 1995b and Shrestha 2000) but still there

\section{Checklist of Birds of Itahari}

Family : Podicipotidae

1. Little grebe

Podiceps ruficollis

Family : Phalacrocoracidae

2. Little cormorant

Phalacrocorax niger

Family : Ardeidae

3. Pond heron

4. Night heron

5. Cattle egret

6. Little egret

7. Intermediate egret

8. Chestnut bittern

Ardeola grayii

Nycticorax nycticorax

Bubulcus ibis

Egretta garzetta

Egretta intermedia

Ixobrychus minutus

\section{Family : Ciconiidae}

9. Open-billed stork

10. Lesser-adjutant stork

Anastomus oscitans

Leptoptilos dubius

11. White-necked stork

Ciconia episcopus

Family : Threskiornithidae

12. Black ibis

Pseudibis papillosa

Family : Accipitridae

13. White-backed vulture

14. Sparrow hawk

15. Black kite

Family : Rallidae

16. White -breasted water hen

Gyps bengalensis

Accipiter nisus

Milvus migrans

migrans

Amaurornis

phoenicurus

Family : Jacanidae

17. Pheasant-tailed Jacana

Hydrophasianus

chirurgus

Family : Charadriidae

18. Red- wattled lapwing

19. Spur-winged lapwing

Vanellus indicus

Vanellus spinosus

Family : Columbidae

20. Spotted dove

21. Indian ring dove

22. Red turtle dove

Streptopelia chinensis

Streptopelia decaocto

Streptopelia

tranquebarica

23. Thick-billed green pigeon Treron curvirostra

24. Blue rock pigeon

Columba livia were some more birds, which I couldn't include in the present checklist.

Family : Psittacidae

25. Rose ringed parakeet Psittacula krameri

Family : Cuculidae

26. Pied crested cuckoo

27. Common hawk-cuckoo

28. Indian koel

29. Large coucal

Clamator jacobinus

Cuculus varius

Eudynamys

scolopacea

Centropus sinensis

Family : Strigidae

30. Barred owlet

31. Spotted owlet

Glaucidium cuculoides

Athene brama

Family : Caprimulgidae

32. Little night jar

Caprimulgus asiaticus

Family : Apodidae

33. House swift

34. Palm swift

Apus affinis

Cypsiurus parvus

Family : Alcedinidae

35. White-breasted kingfisher

36. Eurasian kingfisher

37. Blue-eared kingfisher

38. Lesser pied kingfisher

Halcyon smyrnensis

Alcedo atthis

Alcedo meninting

Ceryle rudis

Family : Meropidae

39. Green bee-eater

40. Blue-tailed bee-eater

Merops orientalis

Merops philippinus

Family : Coraciidae

41. Indian roller

Coracias benghalensis

Family : Upupidae

42. Hoopoe

Upupa epops

Family : Rostratulidae

43. Fantail snipe

Capella gallinago

Family : Capitonidae

44. Crimson- breasted barbet Megalaima

haemacephala

Megalaima asiatica

45. Blue- throated barbet

Megalaima australis 
Our Nature (2003) 1

Family : Picidae

47. Brown- crowned pigmy Dendrocopos nanus woodpecker

48. Larger golden-backed woodpecker

49. Lesser golden-backed woodpecker

Family : Alaudidae

50. Bush Lark

\section{Family : Hirundinidae}

51. Barn swallow

52. Striated swallow

Family : Daniidae

53. Black-headed shrike

54. Rufous-backed shrike

55. Brown shrike

56. Lesser wood shrike

Family : Oriolidae

57. Golden oriole

58. Blackheaded oriole

59. Blacknaped oriole

Family : Dicruridae

60. Black drongo

61 . Ashy or grey drongo

Family : Sturnidae

62. Common myna

63. Grey-headed myna

63. Brahminy myna

\section{Family : Corvidae}

64. House crow

65. Jungle crow

66. Indian Tree pie

Family : Campephagidae

67. Large cuckoo shrike

Family : Pycnonotidae

68. Red-vented bulbul

69. Red -whiskered bulbul

70.White-cheeked bulbul

Family : Muscicapidae

71. Large grey babbler

72. Jungle babbler

\section{Chrysocolaptes \\ lucidus \\ Dinopium benghalense}

Mirafra assamica

Hirundo rustica

Hirundo daurica

Lanius schach tricolor

Lanius schach

Lanius cristatus

Tephrodornis

pondicerianus

Oriolus oriolus

Oriolus xanthornus

Oriolus chinensis

Dicrurus adsimilis

Dicrurus leucophaeus

Acridotheres tristis

Sturnus malabaricus

Sturnus pagodarum

Corvus splendens

Corvus

macrorhynchos

Dendrocitta

vagabunda

Coracina

novaehollandiae

Pycnonotus jocosus

Pycnonotus

leucogenys

Turdoides malcolmi

Turdoides striatus
Pycnonotus cafer
73. Red-breasted flycatcher

74. Verditer flycatcher

75. Grey-headed flycatcher

76. White- throated fantail flycatcher

77. Paradise flycatcher

78. Tailor bird

79. Clamorous reed warbler

80. Dusky leaf warbler

81. Crowned leaf warbler

82. Yellow-eyed leaf

83. Yellow-throated leaf warbler

84. Blue throat

85. Magpie-Robin

86. Black redstart

87. Eurasian rubythroat

88. Collard bush chat

89. Pied bush chat

90. Orange-headed ground thrush

91. Black-throated thrush

92. Red-throated thrush

Family : Paridae

93. Grey tit

Family : Motacillidae

94. Paddyfield pipit

95. Yellow-headed wagtail

96. Pied wagtail greybacked form

97. Pied wagtail black-backed form

Family : Dicaeidae

98. Plaincoloured flowerpecker

Family : Nectariniidae

99. Purple sunbird
Muscicapa parva

Muscicapa

thalassina

Culicicapa

ceylonensis

Rhipidura albicollis

Terpsiphone paradisi

Orthotomus sutorius

Acrocephalus

stentoreus

Phylloscopus

fuscatus

Phylloscopus

reguloides

Seicercus burkii

Phylloscopus

cantator

Erithacus svecicus

Copsychus saularis

Phoenicurus

ochruros

phoenicuroides

Erithacus calliope

Saxicola torquata

Saxicola caprata

Zoothera citrina

Turdus ruficollis

atrogularis

Turdus ruficolli

ruficollis

Parus major

Anthus

novaeseelandiae

Motacilla citreola

Motacilla

maderaspatensis

Dicaeum concolor

Nectarinia asiatica 
Our Nature (2003) 1

Family : Ploceidae

100. House sparrow

101. Baya weaver

102. Whitebacked munia
Passer domesticus Ploceus philippinus Lonchura striata
103. Spotted munia

Family : Emberizidae

104. Yellow-breasted bunting
Lonchura malacca

Emberiza aureola

\section{Acknowledgements}

The author expresses his sincere thanks to Dr. Bharat Raj Subba for extending his

\section{References}

Ali, S. 1989. The Birds of Sikkim. Oxford University Press.

Ali, S. 1990. The book of Indian birds. Oxford University Press.

Ali, S. and S. D. Ripley 1994. A Pictorial Guide to the Birds of Indian subcontinent, Oxford University Press.

helping hand in identification of birds .local people of Itahari are also thankfully acknowledged.

$$
\begin{array}{r}
\text { Manish Raj Pandey } \\
\text { Mahendra Morang Adarsha Multiple Campus, } \\
\text { Biratnagar }
\end{array}
$$

Fleming, R. L. (Sr.), R. L. Fleming (Jr.) and L. S. Bangdel 1984. Birds of Nepal with reference to Kashmir and Sikkim. Nature Himalayas Kathmandu, Nepal.

Shrestha, T. K. 2001. Birds of Nepal, Vol.2, R. K Printers, Kathmandu, Nepal.

Subba, B. R. 1995a. Checklist of Birds of Dharan, Newsletter for Birdwatchers 34 (4): 115-116.

Subba, B. R. 1995b. Checklist of Birds of Biratnagar Newsletter for Birdwatchers 34 (6): 128-129.

\section{Ecological Study of Helminth Parasites in Fishes}

Parasites depend upon other organisms for food and shelter. The relationship between the parasite and the host is termed Parasite Ecology. Parasites choose the host and usually appear fewer hazards than a predator of its prey. However a host suffers from variety of parasites at the same time and also a parasite can use different host for nutrient materials plus shelter.

Helminthes parasite victimizes fishes. Chauhan (1943),Yamaguti (1971), Shrivastava (1985) contributed on fish helminth parasites. To observe fish helminthes parasite in peritoneal cavity and gut, a study was carried out in fishes of Morang and Sunsari districts during July 1994-June 1995, regarding the period of occurrence, incidence and intensity of infection.

During the study 900 fishes of different species were collected and examined freshly as well as in preserved condition. The fishes were cut ventrally to open the body cavity and the parasites were collected carefully. After removing the parasites from the peritoneal cavity (body cavity), gut was cut into several pieces of convenient length and each was vigorously shaken in a dish of warm water. The smaller worms were left at the bottom of the dish. The pieces of gut were then cut open and the mucosa was scraped off into a dish of water to take out cestodes and other smaller worms if present. All the parasites recovered from each fish were collected and counted. The collected 